\title{
Exploiting Voxel Correlation for Automated MRI Bias Field Correction by Conditional Entropy Minimization
}

\author{
Eduard Solanas and Jean-Philippe Thiran \\ Signal Processing Laboratory (LTS) \\ Swiss Federal Institute of Technology (EPFL) \\ CH-1015 Lausanne, Switzerland \\ \{Eduardo.Solanas, JP.Thiran\}@epfl.ch \\ http://ltswww.epfl.ch/ brain
}

\begin{abstract}
An unsupervised model-based strategy for bias field correction is proposed. We assume that information (in the sense of the information theory) in the corrupted image is greater than that in the uncorrupted one. The method exploits the fact that neighboring voxels are highly correlated to correct the bias field using a linear model.
\end{abstract}

\section{Introduction}

Besides noise corruption, deficient brightness of MR images due to radio frequency (RF) field inhomogeneities represents one of the major problems for image analysis. To correct this phenomenon we propose to take advantage from neighboring voxels' information using an extension of Likar's method [1].

\section{Problem Formulation}

Intensity inhomogeneities correction is concerned with finding $\widehat{U}(x, y, z)$, which best estimates the true image $U(x, y, z)$, from the acquired image $N(x, y, z)$

$$
N(x, y, z) \stackrel{\text { correction }}{\longrightarrow} \widehat{U}(x, y, z) \approx U(x, y, z)
$$

$N(x, y, z)$ is usually described by a linear model of image formation (Beckers et al., 1994; Madisetti and Williams, 1998). Therefore, the corrected image can be calculated by inverting the image formation model (2).

$$
N(x, y, z)=U(x, y, z) S_{M}(x, y, z)+S_{A}(x, y, z)
$$

\section{Neighborhood Information for Retrospective Bias Estimation (NIRBE)}

Our method estimates the parameters for our correction model by optimizing a cost function that is a modified first order conditional entropy. Let $X$ denote the 
entire set of symbols $x_{1}, x_{2}, \ldots, x_{M}$ where symbol $x_{i}$ occurs with probability $P_{i}$ and conveys the self-information $I_{i}$, and that satisfy $\sum_{i=1}^{M} P_{i}=1$. If we suppose that a source has a first order dependence, so it is dependent on just their nearest symbols, then the conditional entropy, which represents the average information per symbol is defined as in eq. 3, where $P_{i j}$ is the conditional probability that symbol $x_{i}$ is chosen when its neighbor is symbol $x_{j}$. Averaging over all possible neighbor symbols we obtain the entropy of the source $H(X)$.

$$
H\left(X \mid x_{j}\right) \triangleq-\sum_{i} P_{i j} \log P_{i j} \Rightarrow H(X)=\sum_{j} H\left(X \mid x_{j}\right)
$$

\section{Experimental Results}

For the evaluation of the proposed method we used a set of synthetic data without any pre-processing step. We used the coefficient of variation (cv) and coefficient of joint variations (cjv) to compare the performance between a model which assumes voxels' independence and ours. We assumed a linear model with polynomials of degree 2 for the correction, and the correlation between voxels is represented by the norm of the gradient though the method itself can use any neighborhood information taking into account hardware limitations.

Table 1. Correction of synthetic data using the Entropy $(\mathrm{H})$ and the Conditional Entropy $(\mathrm{CH})$ for information extraction

\begin{tabular}{llllll}
\hline Mod & Bias & Correction & $c v(G M)$ & $c v(W M)$ & $c j v(G M, W M)$ \\
\hline T1 & $0 \%$ & - & 0.17628 & 0.03668 & 0.58053 \\
& $40 \%$ & - & 0.25057 & 0.16058 & 0.876 \\
& $40 \%$ & H & 0.21089 & 0.15199 & 0.88807 \\
& $40 \%$ & CH & 0.23469 & 0.10604 & 0.76078 \\
\hline T2 & $0 \%$ & - & 0.20016 & 0.06213 & 0.95146 \\
& $40 \%$ & - & 0.23691 & 0.22026 & 1.12067 \\
& $40 \%$ & H & 0.23897 & 0.21384 & 1.24981 \\
& $40 \%$ & CH & 0.19955 & 0.22807 & 0.94148 \\
\hline PD & $0 \%$ & - & 0.07473 & 0.01155 & 0.70667 \\
& $40 \%$ & - & 0.12307 & 0.13825 & 1.9651 \\
& $40 \%$ & H & 0.11985 & 0.13655 & 1.94841 \\
& $40 \%$ & CH & 0.12151 & 0.13876 & 1.9972 \\
\hline
\end{tabular}

\section{References}

1. Likar, B., Viergever, M. A., Pernus F.: Retrospective Correction of MR Intensity Inhomogeneity by Information Minimization. Medical Image Computing and Computer-Assisted Intervention (MICCAI 2000), 375-384. 